\title{
Characterisation of Carbonaceous Deposition in Oil Exposed Surfaces at the Nanoscale
}

\author{
Domna-Maria Kaimaki ${ }^{1}$, Ben E. Smith ${ }^{2}$, Sorin Vasile Filip ${ }^{3}$ and Colm Durkan ${ }^{1}$
}

\begin{abstract}
Carbonaceous deposits in oil exposed surfaces are responsible for compromising performance and reducing profitability across the hydrocarbons value chain. In particular, in upstream operation, fouling between the well and the production facility has been found to reduce flow, availability and reliability resulting in lost production. Thus, a better understanding of the processes leading to the deposition of these complex and heavy organic compounds is required, since it is unclear whether they primarily aggregate in the liquid phase or at the liquid-solid interface. In an effort to understand the mechanisms behind deposition, this study uses different modalities of atomic force microscopy (AFM) to characterise relevant metallic, oil exposed surfaces with deposits already on them. More specifically, in this post-mortem analysis, surfaces exposed to oil with and without the presence of an inhibitor are imaged in an effort to pinpoint the effect of the inhibitor on deposition.
\end{abstract}

\section{INTRODUCTION}

During the process of crude oil extraction and subsequent utilisation, changes in the thermodynamic stability of its constituent organic molecules have been observed, caused by external factors such as changes in pressure or temperature. This causes the organic molecules to precipitate out of solution, begin to flocculate and eventually adhere to the surrounding surfaces altering their material properties and eventually leading to fouling [1].

Prior research reveals very little about the mechanisms of aggregation and deposition that occur after the flocculation of the organic molecules and before the fouling of the relevant components [2],[3]. More specifically, the question becomes whether the organic molecules form nanoaggregates and clusters in the liquid phase and then get adsorbed to the surface or whether individual molecules adsorb on the surface, potentially forming a monolayer, and then attract others leading to aggregation at the liquid-solid interface [4].

This is a very challenging problem since the relevant surfaces are subject to a variety of different conditions in terms of their metallurgy, the temperatures and pressures they are exposed to and the composition of the oils they come in contact with. Therefore, in order to generalise the problem,

\footnotetext{
*This work was supported by the BP International Centre for Advanced Materials (BP-ICAM).

1 Domna-Maria Kaimaki and Colm Durkan are at the Nanoscience Centre, University of Cambridge, $11 \mathrm{JJ}$ Thomson Avenue, Cambridge, CB3 OFF, UK

${ }^{2}$ Ben E. Smith is with BP Exploration Operating Company Limited, Chertsey road, Sunbury on Thames, Middlesex, TW16 7BP, UK

${ }^{3}$ Sorin Vasile Filip is with BP Formulated Products Technology, Research \& Innovation Technology Centre, Whitchurch Hill, Pangbourne, Berkshire, RG8 7QR, UK
}

the method chosen to tackle it was a post-mortem top-down approach.

Prior work indicated that the process of deposition is not reversible, leading to chemical changes at the surface. Therefore, simple techniques such as cleaning or scraping the deposits from the relevant surface do not result in the metallic material returning to its initial state. Thus, a variety of fouled surfaces were imaged to characterise the topography of deposits, gain information on their mechanical properties and investigate whether there is any preference in the regions on which these organic molecules are deposited, i.e. whether this is in any way a directed process [5]. This characterisation process was repeated after the surfaces were cleaned to show the irreversibility of the process and characterise the residual deposits that were expected to yield some results on the mechanism behind the initial deposition.

\section{EXPERIMENTAL PROCEDURE}

\section{A. Preparation}

The amount of carbonaceous deposition on the surfaces of the metallic coupons analysed was inhomogeneous. Using an optical microscope as well as looking by eye we could identify three different regions that were called "clean", boundary and "dirty" according to the amount of deposition found there as shown in Fig.1. The coupons were initially characterised as they were received with topographical images taken for each of the representative regions.

Subsequently, we were able to prepare $4 \mathrm{~cm}$ sections of the coupons that could be better mounted in an atomic force microscope (AFM) for full characterisation, while including areas from all the representative regions. Finally, the coupons were cleaned by being immersed in toluene for a minute. The cleaning process was repeated 2-3 times until the residual deposits were no longer visible by eye and the same characterisation process was followed.

\section{B. Methodology}

The morphology of the samples was examined using super-sharp AFM tips with an Al coating on the back-side, and with a nominal resonance frequency of $325 \mathrm{kHz}$ and stiffness of $40 \mathrm{~N} / \mathrm{m}$. Both the amplitude and phase of the cantilever were monitored. It is known that the phase of an oscillating AFM cantilever operating in tapping mode (driven at a frequency slightly below the fundamental resonance) will shift relative to the driving force by an amount proportional to the amount of energy that is dissipated during the tipsample contact [6]. 


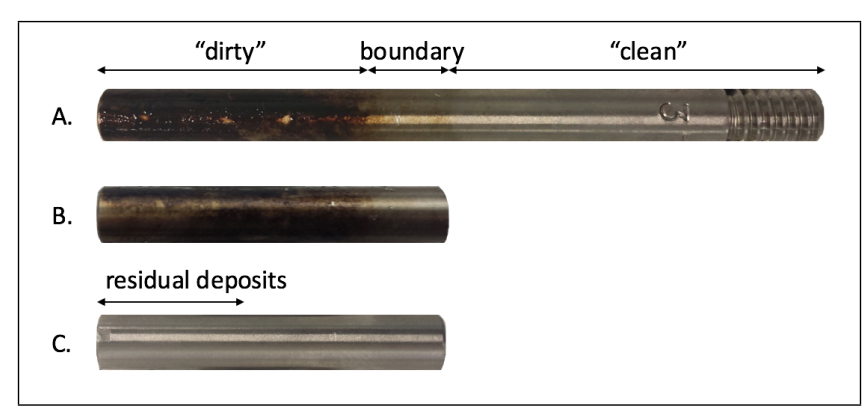

Fig. 1. Images of the coupons in the different stages of characterisation: A. coupon as received, B. section of coupon and C. cleaned coupon

This can be used to distinguish between different materials (albeit qualitatively) and as the dissipation occurs during "contact", phase images tend to display significantly higher spatial resolution than topographic images. We have determined that there is a substantial difference between the phase shift of the cantilever when it is on a deposit as compared to when it is on a bare metal surface. The polarity of the phase shift can tell us something about the interaction between the tip and the sample in that a net attractive force leads to a negative phase shift and a net repulsive force leads to a positive phase shift [7]. Phase imaging is therefore a useful technique to employ when looking at carbonaceous deposits.

In an effort to obtain more quantitative data to help distinguish between the surface and the deposits, the AFM was operated in HybriD mode (Trademark NT-MDT) to map their mechanical properties. In order for the HybriD mode to be successful, the correct contact mechanics model needs to be chosen. When the adhesion force is bigger than the maximum load, the DMT or JKR models are appropriate. We chose the JKR model to map the coupons initially since they were covered by a thick mat of deposits and thus, their surface was soft, and changed to the DMT model after the coupons were cleaned and their surface was stiffer, as indicated in literature[8].

\section{RESULTS AND DISCUSSION}

There were two types of coupons characterised in this study: coupons exposed to oil without an inhibitor and coupons exposed to oil with an inhibitor. Images ranging from a scan size of $45 \mu \mathrm{m}$ to $200 \mathrm{~nm}$ have been acquired for each type of coupon before and after cleaning with toluene.

\section{A. Before toluene}

1) Without inhibitor: Imaging in the so called "clean" region of the sample revealed its inherent topographic features, which comprise regular grooves along the long axis. Moreover, the phase image shows contrast at the location of the edges of the grooves while it is uniform everywhere else indicating that the material has little variation in mechanical properties across the imaged area, justifying the name given to that region.

The "dirty" region, located at the top of the left hand side of Fig. 2, was full of deposits visible as flakes on the surface. A few features of lateral size around $500 \mathrm{~nm}$ to $1 \mu \mathrm{m}$

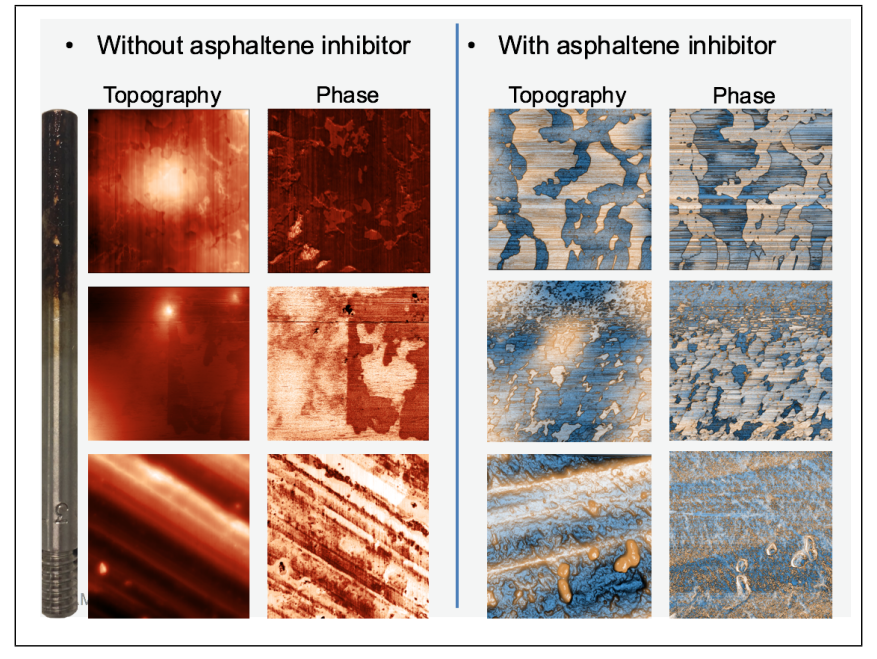

Fig. 2. AFM tapping mode topography and phase images of all representative regions of coupons without inhibitor (scan size: $12.5 \mu \mathrm{m}$ ) and with inhibitor (scan size: $12 \mu \mathrm{m}$ )

and thickness of up to $250 \mathrm{~nm}$ have been found but overall, there is no clear way of knowing the thickness of the mat of deposits in this area.

After examining the two extremes of the sample, the boundary region was imaged in an effort to find an area with thinner deposits. From the topography image in the middle of Fig. 2, we can see a layer of deposits that is 15 $\mathrm{nm}$ thick and appears to be rather uniform. There was also an indication of clusters as well as nanoaggregates however, getting a high resolution image of a small scan size proved to be very difficult because they were highly mobile and thus, moved by the tip.

2) With inhibitor: Similarly to the coupon without inhibitor, imaging in the so called "clean" region of the coupon with inhibitor reveals regular grooves along the long axis due to the manufacturing of the coupon. Moreover, the phase image shows contrast at the location of the edges of the grooves while it is uniform everywhere else indicating that the material has little variation in mechanical properties across the imaged area. Some particles are also found on this region however, the phase shows contrast only around the edges of the particles, indicating that they are probably not organic deposits.

At the boundary region of the coupon, located in the middle of the right hand side of Fig. 2 we find islands of deposits indicated as brighter in the topography image due to the fact that they are higher and darker in the phase indicating stronger attractive interaction between them and the tip. There is a big variation in the size of these islands which could indicate that some are due to clusters (100s of $\mathrm{nm}$ across, $\sim 20 \mathrm{~nm}$ thick) and some due to nanoaggregates (10s of nm across and a few nm thick).

As we move towards the "dirty" region, located at the top of the right hand side of Fig. 2, the deposits grew considerably in size. We zoomed in further to obtain some higher resolution images of the same area as shown in Fig. 3. 
What we see in these images is that there are two types of deposit morphology. The darker areas in topography show deposits that are semi-crystalline as indicated by the grain boundaries whereas the brighter areas show deposits that are amorphous. Moreover, the amophous deposits seem to be more mobile than the semi-crystalline ones, since the tip is having difficulty scanning in those areas, which indicates that there is a stronger interaction between the surface and the deposits than among the deposits.

After observing the images of coupons without and with inhibitor, it seems that the inhibitor is delaying the adsorption of deposits allowing them to form semi-crystalline layers. After that they adhere on the surface and the rest of the deposits seem to grow by forming clusters on top of the initial layers.

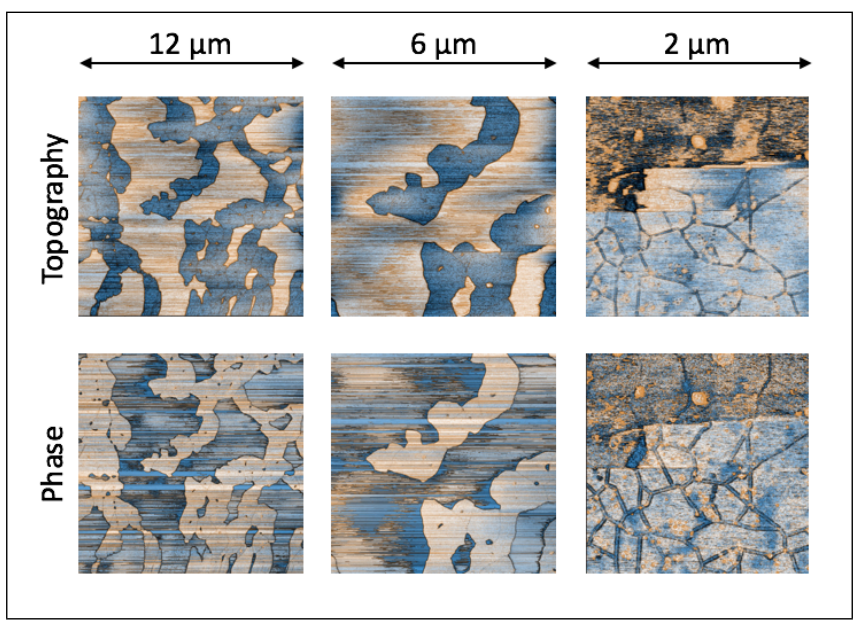

Fig. 3. AFM tapping mode topography and phase images of the "dirty" region of the coupon with inhibitor

\section{B. After toluene}

Once the topography of the coupons with deposits already on them was mapped, an effort was made to mechanically characterise them using HybriD mode. However, this was proven to be very challenging due to the mobility and softness of the deposits even after using the JKR contact model which is the most appropriate one for such samples according to literature [8]. It was then decided to clean the coupons using toluene in an effort to decrease the thickness of the deposits and characterise the residual ones.

1) Without inhibitor: Images of scan sizes ranging from $12 \mu \mathrm{m}$ to $2 \mu \mathrm{m}$ were acquired for the region with residual deposits. Fig. 4 shows the mapping of mechanical properties for an area of $4.5 \mu \mathrm{m}$. In the topography image we observe the regular grooves of the surfaces as well as some deposits. These deposits have a lower elastic modulus than the surface, as expected, and they have a higher deformation since they are softer. However, we expected that the adhesion energy of the deposits would be higher than that of the surface given the strong interaction of the tip with the deposits observed previously from the phase images. One of the causes of the low adhesion energy could be that the tip could be hydrophilic. In an effort to account for the hydrophilicity or hydrophobicity of the tip, functionalised tips will be used in the next stage of experiments.

When it comes to the elastic modulus some further analysis was done to obtain its distribution shown in Fig. 5. The histogram exhibits two peaks: the biggest one is consistent with the elastic modulus of the steel surface $(\sim 200 \mathrm{GPa})$ and the smaller one is $\sim 40 \mathrm{GPa}$, which we can ascribe to the modulus of the deposits observed. This must be taken within the context of the fact that the deposits are a thin (few nm) layer on the steel surface, so this value of Young's modulus is for that coupled system rather than for the carbonaceous deposits on their own, which would be significantly lower.

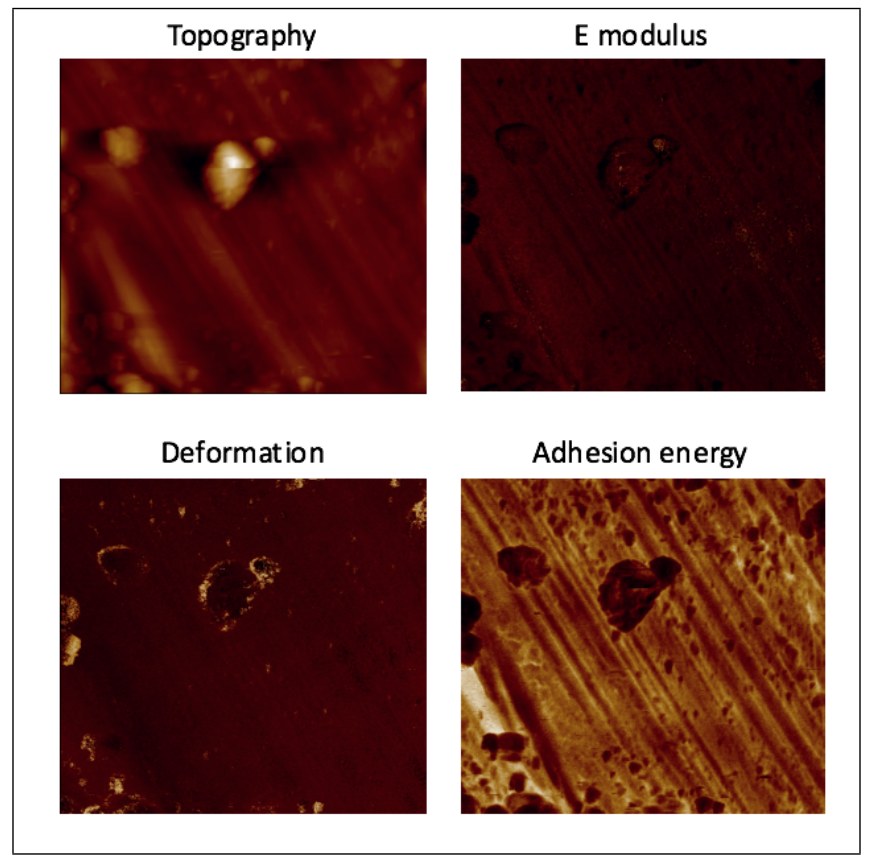

Fig. 4. AFM HybriD mode images of the cleaned coupon without inhibitor. Scan size: $4.5 \mu \mathrm{m}$

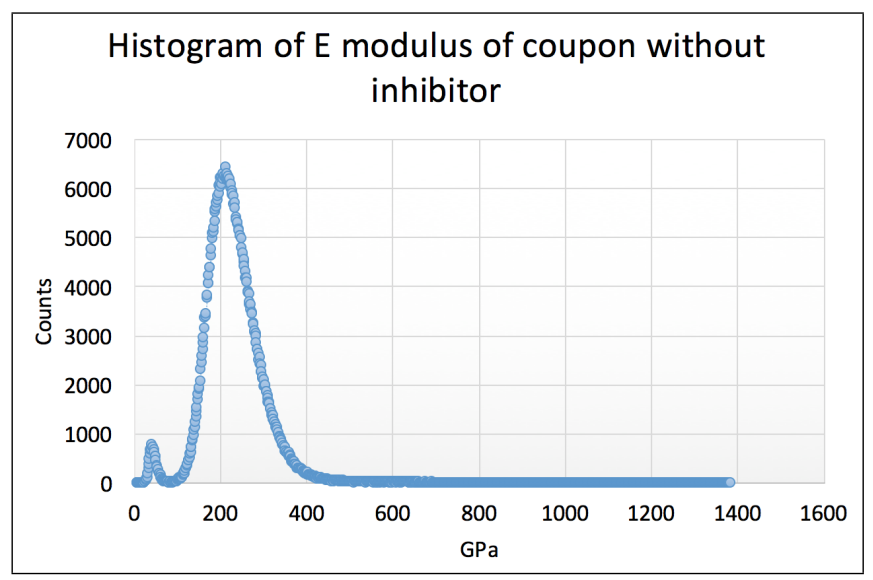

Fig. 5. A histogram of the elastic modulus of the coupon without inhibitor after cleaned.

2) With inhibitor: Similarly to the coupon without inhibitor, the mechanical properties of the cleaned coupon with inhibitor were mapped as shown in Fig. 6. These images 
are considerably more difficult to interpret. The topography shows grooves that are consistent with the manufacturing grooves of the coupon as well as circular clusters. However, it seems that the areas that we would expect to represent the surface are brighter in the image meaning that the topography is higher at these regions. Moreover, the elastic modulus is lower at these areas as well as the stiffness whereas the adhesion energy is higher. All the mechanical properties seem to indicate that these areas are covered by deposits. A preliminary explanation could be that the grooves are indeed covered by deposits as suggested and that the circular clusters are not organic deposits. Further characterisation such as elemental analysis and imaging with functionalised tips is required for conclusions to be made.

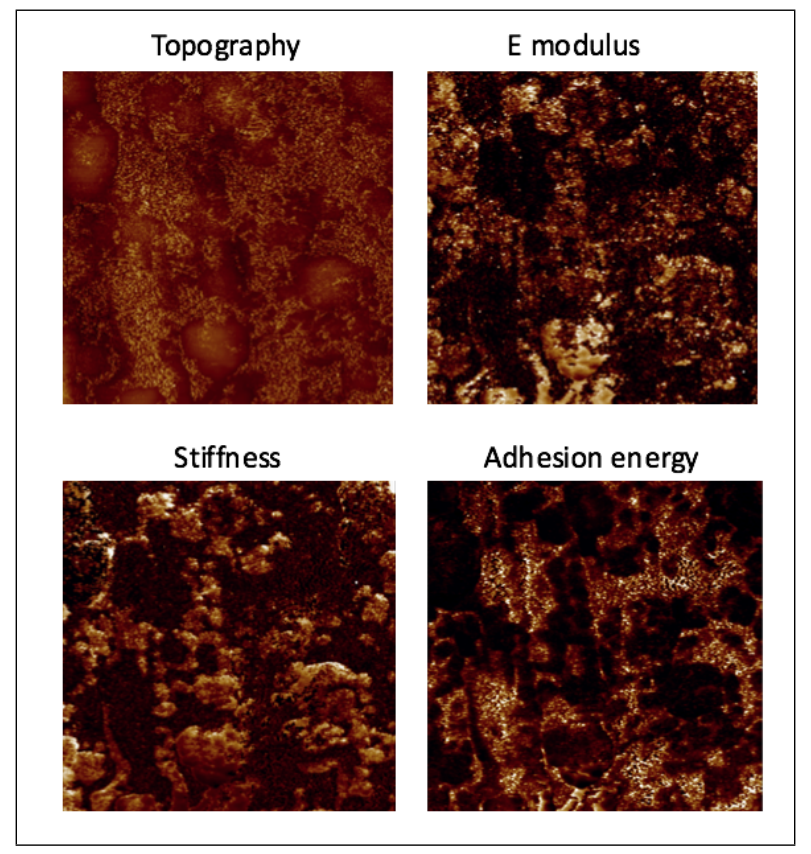

Fig. 6. AFM HybriD mode images of the cleaned coupon with inhibitor. Scan size: $1.5 \mu \mathrm{m}$

\section{CONCLUSIONS}

AFM in topographic and phase mode imaging has been shown to be a useful tool in the characterisation of carbonaceous deposits on metallic surfaces. More specifically, using the aforementioned techniques we were able to show the difference in the nature of deposits with and without an inhibitor. Without an inhibitor, highly mobile deposits were observed in the "dirty" regions and some layers were observed in the boundary regions. However, with inhibitor we were able to observe semi-crystalline deposits forming on the surface and strongly interacting with it as well as weakly bound amorphous deposits on top of them.

However, phase mode imaging is only useful as a qualitative measure of the mechanical properties. Thus, HybriD mode was used to determine the mechanical properties quantitatively and show that even after cleaning the surface is not back to the initial state, i.e. the deposition process is irreversible. The data presented shows consistency with what is expected. Further testing and analysis is required however, in order to make any meaningful conclusions on how the residual deposits shift the mechanical properties of the surface.

\section{ACKNOWLEDGEMENT}

The authors would like to acknowledge the funding and technical support from BP through the BP International Centre for Advanced Materials (BP-ICAM) which made this research possible. They would also like to thank NALCO Champion for suppling the coupons used for this research.

\section{REFERENCES}

[1] N. Arsalan, S. S. Palayangoda, and Q. P. Nguyen, "Characterization of asphaltene deposition in a stainless steel tube," J. Pet. Sci. Eng., vol. 121, pp. 66-77, Sept. 2014.

[2] N. Batina, J. Marzano-Martinez, S. Andersen, and C. Lira-Galeana, "AFM characterization of organic deposits on metal substrates from mexican crude oils," Energy Fuels, vol. 17, pp. 532-542, Mar. 2003.

[3] M. Wang, Y. Hao, M. R. Islam, and C.-C. Chen, "Aggregation thermodynamics for asphaltene precipitaiton," AIChE Journal, Feb. 2016.

[4] B. Schuler, G. Meyer, D. Pena, O. C. Mullins, and L. Gross, "Unraveling the molecular structures of asphaltenes by atomic force microscopy," J. Am. Chem, Soc., vol. 137, pp. 9870-9876, Jul. 2015.

[5] S. Sabbaghi, M. Shariaty-Niassar, S. Ayatollahi, and A. Jahanmiri, "Characterization of asphaltene structure using atomic force microscopy," J. Microsc., vol. 231, pp. 364-373, Jan. 2008.

[6] A. L. Weisenhorn, P. K. Hansma, T. R. Albrecht, and C. Quate, "Forces in atomic force microscopy in air and water," Appl. Phys. Lett., vol. 54, pp. 2651-2653, Jun. 1989.

[7] A. L. Weisenhorn, P. Maivald, H.-J. Butt, and P. K. Hansma, "Measuring adhesion, attraction, and repulsion between surfaces in liquids with an atomic-force microscope," Phys. Rev. B, vol. 45, pp. 226-232, May 1992.

[8] H.-J. Butt, B. Cappella, and M. Kappl, "Force measurements with the atomic force microscope: Technique, interpretation and applications," Surf. Sci. Rep., vol. 59, pp. 1-152, Aug. 2005.

[9] J. Carmichael and D. Fouchard, "New method for the assessment of asphaltene deposition behavior." The 15th International Conference on Petroleum Phase Behavior and Fouling, Jun. 2014.

[10] J. Bae, D. Fouchard, S. Garner, and J. Macias, "Advantages of applying a multifaceted approach to asphaltene inhibitor selection." Offshore Technology Conference, May 2016. 